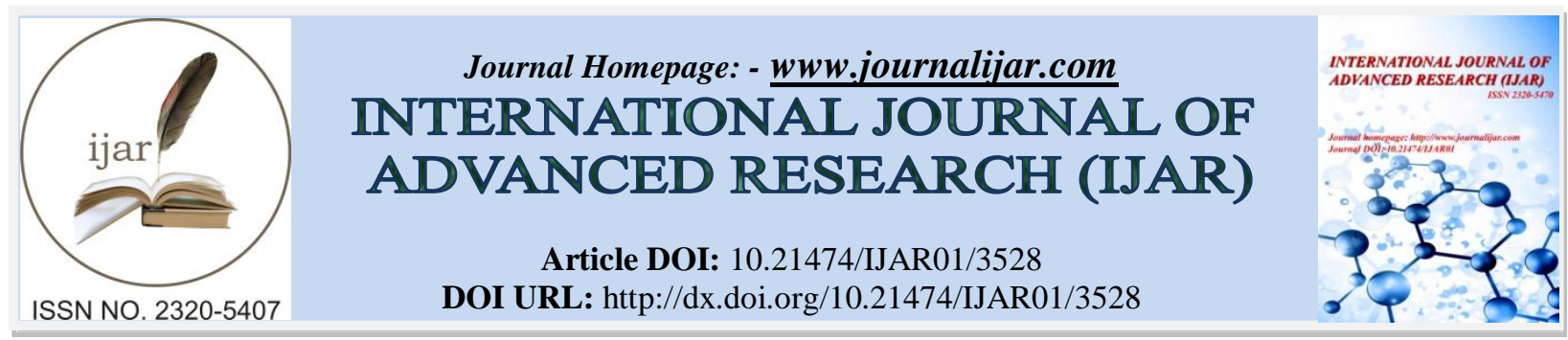

RESEARCH ARTICLE

\title{
IMMEDIATE REATTACHMENT OF FRACTURED TOOTH FRAGMENT USING PRE FRABRICATED GLASS FIBER POST IN A MAXILLARY CENTRAL INCISOR - A CASE REPORT.
}

Dr. Harshal Patel, Dr. Kailash Attur, Dr. Kamal Bagda and Dr. Chinmay Patel.

Narsinhbhai Patel Dental College and Hospital,Visnagar, Mahesana, Gujarat.

\section{Manuscript Info}

Manuscript History

Received: 09 January 2017

Final Accepted: 10 February 2017

Published: March 2017

Key words:-

Crown fractures, fiber

Post, reattachment

\section{Abstract}

Anterior crown fractures are a common form of injury that mainly affects children and adolescents due to their position in the oral cavity especially in the maxillary arch. Reattachment of original tooth fragment to the fractured tooth forms a relatively quick, biologic and esthetic restoration. 32 years old male patient reported to the Department of conservative Dentistry \& Endodontics with a crown fracture of maxillary left central incisor and mid root fracture of maxillary left lateral incisor one day after the trauma had occurred. This case report represent the maxillary left central incisor treated with reattachment of natural crown fragment using glass fiber post and dual core composite resin and in maxillary left lateral incisor treated with endodontic therapy.

Copy Right, IJAR, 2017,. All rights reserved.

\section{Introduction:-}

Dental fractures account for $26-76 \%$ of dental injuries in the permanent dentition. Coronal fractures of permanent incisors represent $18-22 \%$ of all trauma to dental hard tissues, $28-44 \%$ being simple (enamel and dentin) and 11$15 \%$ complex (enamel, dentin, and pulp).Traumatized anterior teeth require quick functional and esthetic repair. This is particularly true in case of young patients as it not only causes physiologic impairment, but also esthetic disfigurement leading to a psychological impact. ${ }^{1}$

Primary goal of the treatment of traumatically injured teeth remains esthetic and functional rehabilitation. Several therapeutic procedures are available to restore fractured anterior teeth. Reattachment of the fractured fragment is an excellent biological approach for restoration, when the fragment is available. ${ }^{2}$

The concept of reattachment began in 1964 when Chosak and Eidelman used a cast post and conventional cement to reattach an anterior crown segment. Recent developments in restorative materials, placement techniques, and adhesive protocols allow reattachment using resin based composites. ${ }^{3}$ Tennery was the first to use acid etch technique for the reattachment of fractured tooth fragment.

With the recent improvements in resin based restorative materials, tooth colored fiber posts along with resin luting cement are of choice because of several advantages such as esthetics, bonding to tooth structure and low modulus of elasticity similar to that of dentin. In some cases where the fracture extends subgingivally, but is supra alveolar, a full-thickness envelope flap provides the required isolation and visibility. ${ }^{1}$ 
This is a case of a permanent incisors with crown and middle root fracture treated using glass fiber post in left maxillary central incisor and Root canal treatment in maxillary left lateral incisor.

\section{Case Report:-}

A 32-year-old male patient reported to the Department of Conservative Dentistry and Endodontics with fractured maxillary anterior tooth. The tooth had fractured 1day ago on account of a minor two wheeler accident. His medical history was noncontributory. Patient complained of severe pain and swelling with 21 \& 22 . On clinical and radiographic examination, it was diagnosed as a complicated crown fracture of the maxillary left central incisor and crown root fracture in maxillary left lateral incisor (Figure-1). The oblique fracture line was supragingival both labially and palatally with 21.The treatment plan decided for the patient was reattachment of the fragment with 21 and root canal treatment in realtion 22 .

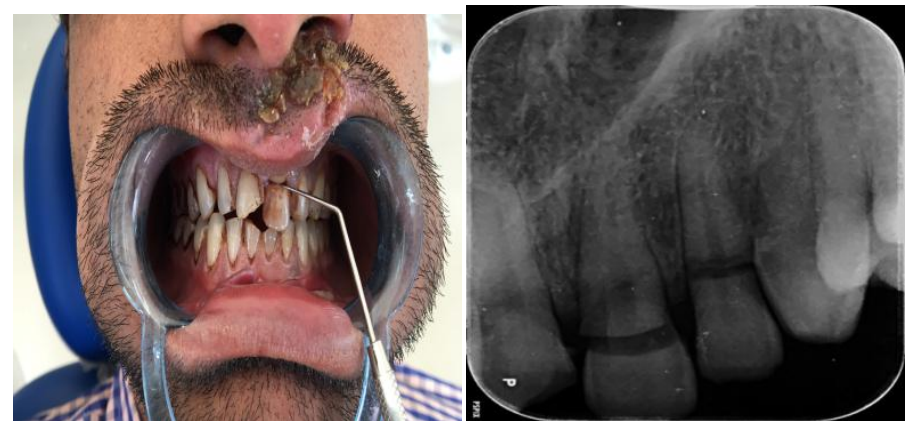

Figure 1:- Ellis class III fracture with 21 and middle root fracture with 22.

Patient was informed of long term prognosis of the teeth and the treatment was initiated.The teeth were anaesthetized using locaI infiltration technique (2\% lidocaine with 1:100,000). Single visit root canal treatment of 21 and 22 was carried out (figure-2). The post space was prepared by using peeso reamers retaining $5 \mathrm{~mm}$ of apical gutta percha and No.2 prefabricated glass fiber post was selected. After checking the fit of the post, it was trimmed leaving about $2 \mathrm{~mm}$ of post beyond the tooth with 21. The pulpal aspect of the fractured fragment was prepared using a straight fissure bur (internal groove).The post and the fragment were adjusted until the fragment was approximated to its original position to obtain retention for the fragment. The post was luted using dual core resin luting cement ( RelyX ${ }^{\mathrm{TM}}$ U200, 3M ESPE, Germany) with 21 [Figure-3].

Both the fragment and tooth were etched with $37 \%$ phosphoric acid etchant gel for 15 seconds. Then the bonding agent (3M ESPE, USA) was applied and light cured for 10 seconds. Flowable composite resin (Filtek Z 350XT flowable restorative,3M ESPE,USA) was applied to both fragment and tooth surfaces. The fractured segment was then accurately attach on the tooth, paying special attention to the fit between the segments. When the original position had been reestablished, excess resin was removed and the bonded area was light cured for 40 seconds on each surface.

The occlusion was carefully checked and adjusted. The margins were properly finished with finishing burs and polished with polishing paste. Final radiograph was taken to confirm the proper alignment of the attached fragment. The patient was given oral hygiene instructions and was recalled for follow-up visits. The six month follow up (Figure-4) shows satisfactory result esthetically and functionally. 


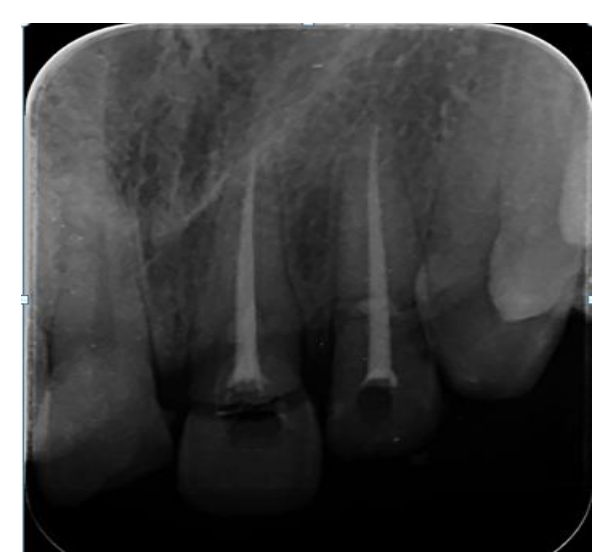

Figure 2:- Root canal treatment was carried out with 21 and 22.

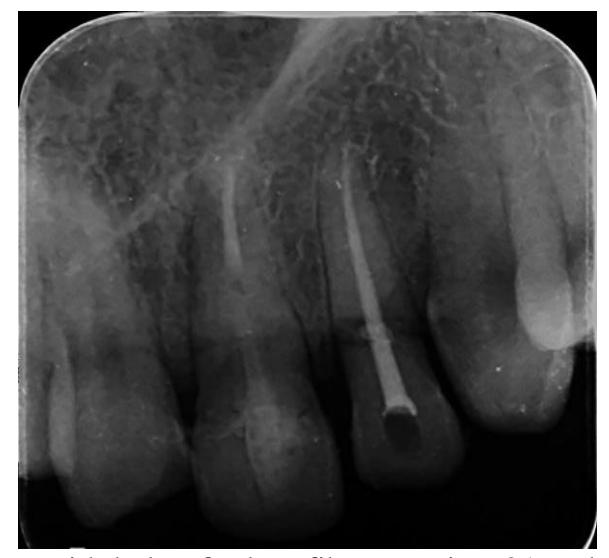

Figure 3:- Reattachment with help of glass fiber post i.r.t.21 and MTA Fillapex i.r.t. 22.

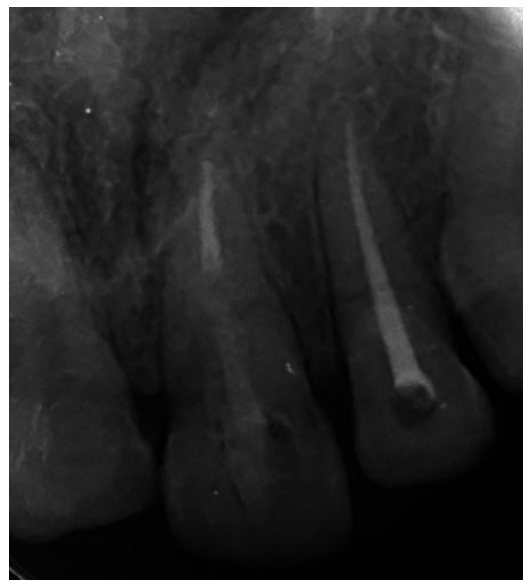

Follow up after 6 months

\section{Discussion:-}

Traumatic dental injuries in the permanent dentition necessarily involve function, aesthetics and psychological aspects particularly in young patients. Factors determining the treatment plan and prognosis for coronal teeth fractures include the extent of fracture, endodontic involvement, periodontal status, maturity of root, biological width invasion, presence / absence of fractured tooth fragment and the fit of the fragment and remaining tooth, occlusion, esthetics and finances. Depending on these factors, various treatment options are composite restorations, orthodontic / surgical extrusion, crown lengthening followed by post and core supported restorations and reattachment of fractured fragment. ${ }^{2,4}$ 
Conventional methods employed in the restoration of fractured teeth include partial and full coverage crowns, laminate veneers, and composite resins all of which are time consuming, high priced, and not conservative. ${ }^{4}$ It is beneficial to quickly restore the function and the aesthetics of a fractured tooth. The reattachment of a fractured crown fragment may be the most conservative and desirable treatment of choice for anterior teeth. The reattachment of the crown fragment to a fractured tooth is the best method to reinstate the natural shape, contour, surface texture, occlusal alignment and color of the fragment, which offers excellent esthetic and functional result. ${ }^{5}$

Post and core supported crowns are recommended in cases of major tooth loss where the fractured fragment is not available. With the fracture line extending below the alveolar crestal bone orthodontic extrusion or surgical extrusion is recommended before the restoration. But with the fracture lying above the alveolar bone crest reattachment of the fractured fragment is a more viable option. There is sufficient evidence to show that the reattachment of the fractured coronal fragments using modern adhesive systems results in predictably successful short- and medium-term outcomes. ${ }^{1}$

However, this technique can be used only when the intact tooth fragment is available. As the fractured fragments were intact in the cases described above, use of natural tooth substance clearly eliminated problems of differential wear of restorative material unmatched shades and difficulty of contour and texture reproduction associated with other restorative techniques. ${ }^{7}$

The common complications in the post and core system are debonding and root fracture. These are more common with cast metal posts used earlier which can cause wedging forces coronally that may result in irreversible failure because of fracture of an already weakened root. The newer variety of nonmetallic posts is made of either ceramic or fiber reinforced materials like carbon, quartz or glass in an epoxy matrix. Fiber reinforced composite resin post offers many advantages for reattachment procedure such as conservation of tooth structure, bonding to the tooth structure, low modulus of elasticity equal to that of dentin, esthetics, simplicity of procedure requiring less chair time and cost-effectiveness. ${ }^{8}$

By using glass fiber post with composite core and with recent advances in adhesive techniques and materials one can create a Monoblock, a multi-layered structure with no inherent weak interlayer interfaces. Early retrospective studies indicate that the clinical performance of fiber post is promising and the failure rate recorded is $3.2 \%$ over a period of up to 4 years. Trope et al. in 1985 showed that Endodontically treated teeth can be reinforced with the use of resin composite restoration. The flowable composite reinforces the tooth, helps in achieving higher bond strengths and minimizes the inclusion of air voids. ${ }^{1}$

Tooth fracture reattachment allows restoration of the tooth with minimal sacrifice of the remaining tooth structure. The use of natural tooth substance clearly eliminated problems of differential wear of restorative material, unmatched shades and difficulty of contour and texture reproduction associated with other restorative techniques. ${ }^{2}$

\section{Conclusion:-}

Reattachment technique is the most conservative and biological method of restoring a fractured anterior tooth. Reattaching a tooth fragment with newer adhesive materials may be used successfully to restore fractured teeth with adequate strength, but long term follow up is necessary in order to predict the durability of the tooth-adhesivefragment complex. Patient cooperation and understanding of the limitations of the treatment is of utmost importance for good prognosis. The need of the day is to educate the population to preserve fractured segment and seek immediate dental treatment. 


\section{References:-}

1. Hegde S G., Tawani G S, Warhadpande M M., Use of quartz fiber post for reattachment of complex crown root fractures : A 4-year follow up. JCD 2014;17(4): 389-392.

2. Jyothi M, Jyothirmayi B SL, Sirisha K, Mounika A, Girish K, Sruthi Keerthi M H, Reattchment - Conservative management of complicated crown fracture in anterior teeth. IJAD 2016;2 (1):10-13.

3. Choudhary A, Garg R, Bhalla A, Khatri R; Tooth fragment reattachment : An esthetic and biological restoration. Journal of Natural Science, Biology and medicine 2015;6(1): 205-208.

4. Kavitha T. , Laxmi Narayanan L.; Reattachment of fractured tooth fragments using a custom fabricated dowel Three case reports. Endodontology 2000; (12):65-70.

5. Singla M G, Mittal R, Singla A; Tooth reattachment : An immediate esthetic restorative procedure for crown root fracture. Saudi endodontic journal 2012;2(1): 36-40.

6. Khatri A, Kumar S, Kalra N, Tyagi R.Fragments reattachment of a complicated crown-root fracture in primary maxillary central incisor and 1 year follow up. Journal of Research in Dental sciences 2016; (2):124-127.

7. Khatri A, Kumar S, Kalra N, Tyagi R.Reattachment of a vertical complicated subgingival crown root fracture in a 10 year old child : A case report. International Journal of clinical pediatric dentistry 2009;2(3):53-59.

8. Martos J, Majzoob Y M, Signori C, Silveira L F. Adhesive crown fragment reattachment in anterior-fractured tooth. Journal of restorative dentistry 2014;2(1):54-56.

9. Sapna C. M., Priya R., Sreedevi N. B.,Rajan R, KumarR.Reattachment of Fractured Tooth Fragment with Fiber Post: A Case Series with 1-Year Followup. Case Reports in Dentistry 2014.1-5

10. Qureshi A, Kumar P, Rao P S, Soujanya E, Ramtheerth A. Reattachment of coronal fragment using fiber reinforced post. IJMD 2015;4(1):661-665. 